\title{
Spatial learning deficits are not solely due to cholinergic deficits following medial septal lesions with colchicine
}

\author{
STANLEY BARONE, JR. \\ Laboratory of Molecular and Integrative Neuroscience \\ National Institute of Environmental Health Sciences, Research Triangle Park, North Carolina \\ and East Carolina University School of Medicine, Greenville, North Carolina \\ KEVIN P. NANRY and WILLIAM R. MUNDY \\ Laboratory of Molecular and Integrative Neuroscience \\ National Institute of Environmental Health Sciences, Research Triangle Park, North Carolina \\ JACQUELINE F. MCGINTY \\ East Carolina University School of Medicine, Greenville, North Carolina \\ and \\ HUGH A. TILSON \\ Laboratory of Molecular and Integrative Neuroscience \\ National Institute of Environmental Health Sciences, Research Triangle Park, North Carolina
}

\begin{abstract}
Colchicine was infused bilaterally into the cerebrolateral ventricles $(3.75 \mu \mathrm{g} / \mathrm{side})$ or directly into the medial septum ( $5 \mu \mathrm{g})$ of adult, male Fischer-344 rats $(n=48)$, and effects on behavior and cholinergic markers were determined. Rats receiving intracerebroventricular (ICV) administration of colchicine were hyperaggressive during the first week after administration and were hyperactive when tested during 60-min sessions at weekly intervals during the first 3 weeks after colchicine treatment. ICV colchicine also interfered with the acquisition of a spatial task in the water maze. Rats receiving colchicine directly into the medial septum were also aggressive and hyperactive, but were not impaired in the acquisition of the water-maze task. It was subsequently found that direct administration and ICV administration of colchicine both decreased the number of choline acetyltransferase (ChAT) immunoreactive cells in the medial septum by at least $50 \%$ of vehicle-treated rats and decreased ChAT enzyme activity in both the right and the left hippocampus to about $50 \%$ of control levels. However, the acetylcholinesterase (AChE) staining was more clearly decreased bilaterally in the hippocampus after ICV infusion than after direct infusion of colchicine. ICV infusion resulted in bilateral shrinkage of the lateral septum, whereas direct septal administration resulted in only unilateral damage to the lateral septum. The results of these experiments do not support the generally accepted hypothesis that spatial learning deficits seen in animals with medial septum lesions are solely due to a lesion-associated cholinergic deficit in the hippocampus.
\end{abstract}

Recent research has revealed considerable detail concerning the anatomy of forebrain cholinergic pathways (Fibiger, 1982). The cerebral cortex receives cholinergic afferents from cell bodies located in the area of the nucleus basalis, whereas the hippocampus receives afferents from cell bodies located in the medial septum (Johnston, McKinney, \& Coyle, 1979; Lehman, Nagy, Almadja, \& Fibiger, 1980). Data from humans indicate that degeneration of forebrain cholinergic neurons is associated with

The editorial assistance of Loretta Moore in the preparation of this manuscript is gratefully acknowledged. Correspondence should be addressed to Stanley Barone, Jr., Neurotoxicology Division/EPA, MD74B, Research Triangle Park, NC 27711. cognitive dysfunction, particularly in the case of senile dementia of the Alzheimer's type (SDAT) (Whitehouse et al., 1982). Experiments with animals have shown that lesions in the septum and nucleus basalis alter motor activity, learning and memory, and reactivity (Bartus et al., 1985; Gray \& McNaughton, 1983; Knowlton, Wenk, Olton, \& Coyle, 1985; Tilson et al., 1988).

The neurons in the medial septum are known to project to the hippocampus (Bigl, Woolf, \& Butcher, 1982; Swanson \& Cowan, 1979); about $50 \%$ of those neurons are cholinergic (Wainer, Levey, Rye, Mesulam, \& Mufson, 1985; Woolf, Eckenstein, \& Butcher, 1984). In addition, the medial septum contains GABAergic and other types of neurons (Kohler, Chan-Palay, \& Jang-Yen, 1984). To what extent noncholinergic neurons contribute to the be- 
havioral effects of septal lesions is not fully understood, although GABA-containing neurons in the septum control interneurons in the hippocampus (Freund \& Antal, 1988). Regardless, it is generally accepted that interruption of the fimbria-fornix, which connects the septum to the hippocampus and the hippocampus to the septum, reliably interferes with learning and memory of spatial tasks (Becker, Walker, \& Olton, 1980; Nilsson, Shapiro, Olton, Gage, \& Bjorklund, 1987; Olton, Walker, \& Gage, 1978; Sutherland \& Rodriguez, 1989).

Deficits in spatial memory have been observed using the eight-arm radial maze (Becker et al., 1980; Crutcher, Kesner, \& Novak, 1983; Miyamoto, Kato, Narumbi, \& Nagaoka, 1987) and the Morris water maze (Hagan, Salamone, Simpson, Iversen, \& Morris, 1988; Kelsey \& Landry, 1988; Miyamoto et al., 1987; Nilsson, Strecker, Daszuta, \& Bjorklund, 1988). Lesions in the medial septum also reduce markers of cholinergic fibers in the hippocampus (Hagan et al., 1988; Kelsey \& Landry, 1988; Miyamoto et al., 1987; Nilsson et al., 1988), suggesting that the effects of medial septal lesions on learning and memory are associated with decreased cholinergic hippocampal function. In studies investigating the effects of medial septal lesions on acquisition of the Morris water maze, various lesion methods were used, including electric current (Kelsey \& Landry, 1988; Miyamoto et al., 1987), radio frequency (Nilsson et al., 1988), and ibotenic acid (Hagan et al., 1988). However, each of these procedures may produce damage to adjacent cell bodies or axons passing through the medial septum and/or disrupt other fibers projecting through the fimbria-fornix. The purpose of the present experiment was to study the effects of medial septal lesions on acquisition in the Morris water maze using colchicine, a neurotoxicant having no excitotoxicity. Peterson and McGinty (1988) reported that unilateral infusion of colchicine into the lateral cerebroventricles of rats had a relatively specific effect on cholinergic neurons in the medial septum, while sparing other (i.e., GABA) neurons in that area. Our experiment shows that lateral ventricular infusion of colchicine interferes with acquisition in the Morris water maze. However, the direct administration of colchicine into the medial septum had no effect on learning.

\section{METHOD}

\begin{abstract}
Animals
Male Fischer-344 rats (Charles River Breeders, Inc., Raleigh, $\mathrm{NC}$ ), each weighing approximately $275-375 \mathrm{~g}$, were used in all experiments. The animals were housed separately in a colony room controlled for temperature $\left(22^{\circ} \pm 2^{\circ} \mathrm{C}\right)$ and humidity $(50 \% \pm 10 \%)$, on a 12:12-h light:dark cycle (lights on $0700 \mathrm{~h}$ ). Food (NIH Diet No. 31) and water were freely available in the home cage.
\end{abstract}

\section{Septal Lesions}

Rats were anesthetized with sodium pentobarbital (50 mg/kg, i.p.) and positioned in a stereotaxic instrument. A sagital incision was made in the scalp and a hole was drilled through the skull to allow for the insertion of an injection needle. Colchicine was then injected directly into the medial septum. Colchicine $(5.0 \mu \mathrm{g} / 1.0 \mu \mathrm{l})$ or ve- hicle (artificial cerebrospinal fluid, ACSF) was infused over a period of $5 \mathrm{~min}$, and the needle was left in place for an additional $5 \mathrm{~min}$ to allow for maximal diffusion. The coordinates for the direct administration in reference to bregma were incisor bar, $-0.9 \mathrm{~mm}$, $0.7 \mathrm{~mm}$ anterior, $2.5 \mathrm{~mm}$ lateral, and $6.7 \mathrm{~mm}$ at $22.5^{\circ}$ from vertical toward the center of the brain. To infuse colchicine or ACSF into the lateral cerebroventricles, two holes were drilled into the skull and colchicine $(3.75 \mu \mathrm{g} / 2.5 \mu \mathrm{l}$ per side) or ACSF was injected bilaterally into the ventricle at the following coordinates from bregma: incisor bar, $-0.9 \mathrm{~mm}, 0.8 \mathrm{~mm}$ posterior, $1.2 \mathrm{~mm}$ lateral, and $4.2 \mathrm{~mm}$ vertical (Figure 6.1). All injections were made with a Kopf microinjector. The doses of colchicine and coordinates for injections were determined in several pilot studies.

\section{Behavioral Tests}

All animals $(n=48)$ were tested in motor activity chambers and the water maze. Motor activity was measured using four identical Plexiglas chambers $(43 \times 21 \times 18 \mathrm{~cm})$ equipped with two parallel rows of photocells, 20 per side positioned along the long axis of the chamber at 5.5 and $13.5 \mathrm{~cm}$ above the floor. Interruption of a photobeam (one activity count) was recorded and stored for later analysis. Each rat received a single test session 1, 2, and 3 weeks after surgery. Activity was measured at the same time of day during each 60 -min session.

One week after the last locomotor activity measurement (4 weeks after surgery), the acquisition of a spatial navigation task was examined using a water-maze task (Mundy \& Tilson, 1988). Briefly, the animals were trained to swim to a platform hidden in a large circular pool $(148 \mathrm{~cm}$ in diameter $\times 60 \mathrm{~cm}$ in height) located in a test room containing numerous extramaze cues. The pool was filled with water $\left(28^{\circ} \pm 2^{\circ} \mathrm{C}\right)$ to a depth of $40 \mathrm{~cm}$, made opaque with powdered milk. A transparent platform, $10 \mathrm{~cm}$ in diameter and submerged $1.5 \mathrm{~cm}$ below the water surface, was used. Four equally spaced locations around the edge of the pool were employed as start points and divided the pool into four quadrants. The animals received daily sessions consisting of four trials. On each day, all four starting positions were used once in a random sequence without replacement. A trial began when the rat was placed into the maze facing the wall of the pool at one of the starting points. The latency to find the escape platform was recorded up to a maximum of $60 \mathrm{sec}$. If a rat did not escape onto the platform within that time, it was placed on the platform where it remained for $15 \mathrm{sec}$. For each rat, the platform was fixed in the center of one of the four quadrants and remained in that location for the duration of the experiment. There was approximately $5 \mathrm{~min}$ between trials. To determine the extent of spatial learning, the final test day consisted of one 60-sec posttraining retention free-swim trial, during which the platform was removed from the pool. The time spent swimming in each of the four quadrants was recorded for each rat.

\section{Neurochemistry}

At the conclusion of the experiment, 5 weeks after colchicine treatment, the rats were randomly selected and their brains prepared for either neurochemical or neuroanatomical assessment.

The animals ( $n=6$ per treatment) were sacrificed by decapitation, and the brains removed and dissected according to the method of Glowinski and Iversen (1966). The frontal cortex, left and right hippocampus, and septum were stored at $-70^{\circ} \mathrm{C}$ for assay at a later time. The regions were assayed for choline acetyltransferase (ChAT) using the method of Fonnum (1975). Briefly, tissues were

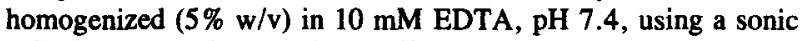
cell disrupter. Triton X-100 $(0.5 \% \mathrm{v} / \mathrm{v})$ was added to homogenates to ensure release of enzyme. Six microliters of a substrate mixture of $0.2 \mathrm{mM}$ C14-acetyl-CoA $53 \mathrm{mCi} / \mathrm{mmol}$ (New England Nuclear, Boston, MA), $300 \mathrm{mM} \mathrm{NaCl}, 50 \mathrm{mM}$ sodium phosphate buffer, pH 7.4, $8 \mathrm{mM}$ choline bromide, $20 \mathrm{mM}$ EDTA, pH 7.4, and $0.1 \mathrm{mM}$ physostigmine were added with $2 \mu$ l of homogenate in a 
microtube and incubated at $37^{\circ} \mathrm{C}$ for $15 \mathrm{~min}$. The microtube was placed in a scintillation vial, washed out with $5 \mathrm{ml}$ of sodium phosphate buffer, followed by $3.2 \mathrm{ml}$ of acetonitrile containing $16 \mathrm{mg}$ of tetraphenylboron, and then $10 \mathrm{ml}$ toluene containing $0.42 \mathrm{ml}$ of PPO-POPOP was added. The vials were shaken lightly for $1 \mathrm{~min}$ and allowed to sit for $15 \mathrm{~min}$ before we counted them in a Beckman scintillation counter. Protein determination was performed with a modification of the assay of Bradford (1976) using a commercially availabie kit (Bio-Rad, Richmond, CA).

\section{Neuroanatomical Assessment}

The remaining animals ( $n=6$ per treatment) examined histologically were sacrificed with an overdose of Nembutal $(100 \mathrm{mg} / \mathrm{kg})$ and perfused with $100 \mathrm{ml}$ of physiological saline, followed by $300 \mathrm{ml}$ of chilled $4 \%$ paraformaldehyde and $0.15 \%$ picric acid in phosphate buffered saline (PBS). The descending aorta was clamped so as to limit the total volume of fixative required. The brains were removed and post-fixed for $1.5 \mathrm{~h}$ in the same fixative before being transferred to $15 \%$ buffered sucrose for cryoprotection. Frozen sections at $50 \mu \mathrm{m}$ were cut coronally in a one-out-of-four series and collected in sodium acetate buffer $(\mathrm{pH}=6.0$ ) or PBS with $0.1 \%$ sodium azide through the septal and hippocampal regions. Adjacent sections saved in PBS were stained with $0.1 \%$ thionin or stained for ChAT immunocytochemistry with a rat monoclonal antibody (Boehringer-Manneheim; Eckenstein \& Thoenen, 1982). The ChAT antibody was diluted 1:200 in PBS with $0.01 \%$ sodium azide; subsequent incubations in this primary antibody were performed for 5 days at $4^{\circ} \mathrm{C}$. Following four rinses in PBS, sections were incubated in biotinylated goat anti-rat antiserum. Sections were again rinsed in PBS and incubated in avidin-biotin immunoperoxidase complex following the methods of Hsu, Raine, and Fanger (1981). The peroxidase complex was visualized by exposing sections to the $(0.05 \%)$ $3,3^{\prime}$-diaminobenzidine (Sigma, MO) in the presence of $(0.003 \%$ ) hydrogen peroxide. Control sections for immunocytochemistry were randomly selected from each treatment and processed under the same conditions as above except that primary antibody was omitted. Under these conditions, peroxidase reaction was observed only in sections of brains receiving direct administration into the medial septum. These sections had a consistent endogenous peroxidase activity ipsilateral to the injection and localized primarily around the vasculature.

Counting of ChAT immunoreactive (IR) cell bodies with nuclear profiles in the medial septum of 5 rats from each treatment was performed on three corresponding sections in the caudal septum, one through the injection site and $200 \mu$ rostral and caudal to this site. The three rostrocaudal levels 600,400 , and $200 \mu \mathrm{m}$ anterior to the decussation of the anterior commissure were examined since this region has the highest density of neurons projecting to the hippocampus (Peterson, 1989). Counting of ChAT IR cell bodies was performed with an Olympus light microscope utilizing a $10 \times$ objective. The right and left medial septum were counted separately, and the data collection was limited to the area superior to an imaginary horizontal line drawn ventrally between the anterior commissures.

In addition, sections from the above animals, saved in sodium acetate buffer, were processed for AChE histochemistry according to the method of Hedreen, Bacon, and Price (1985).

\section{Statistics}

Water-maze and locomotor activity data were analyzed for overall significance using a repeated measures analysis of variance (ANOVA) followed by a Greenhouse-Geisser adjustment for degrees of freedom for all repeated measures factors and interactions with repeated measurement factors to compensate for the lack of sphericity (Geisser \& Greenhouse, 1958; Greenhouse \& Geisser, 1959). Post hoc comparisons between treatment groups were made using Fisher's least significant difference test. Data from the posttraining free-swim test in the water maze and the neurochemical data were analyzed using a Student's $t$ test. Data from cell counts were analyzed using a threeway ANOVA, with treatment (colchicine vs. ACSF), method of treatment (ICV vs. direct administration), and side (right vs. left) as the variables.

\section{RESULTS}

\section{Locomotor Activity}

Colchicine administered directly into the medial septum or via the cerebrolateral ventricles produced hyperactivity and aggressiveness during the first week after surgery, effects that diminished gradually by 3 weeks after surgery. Both the direct administration and the intracerebroventricular (ICV) injection of colchicine increased locomotor activity 1,2, and 3 weeks after injection (Figure 1). A repeated measures ANOVA of the data from

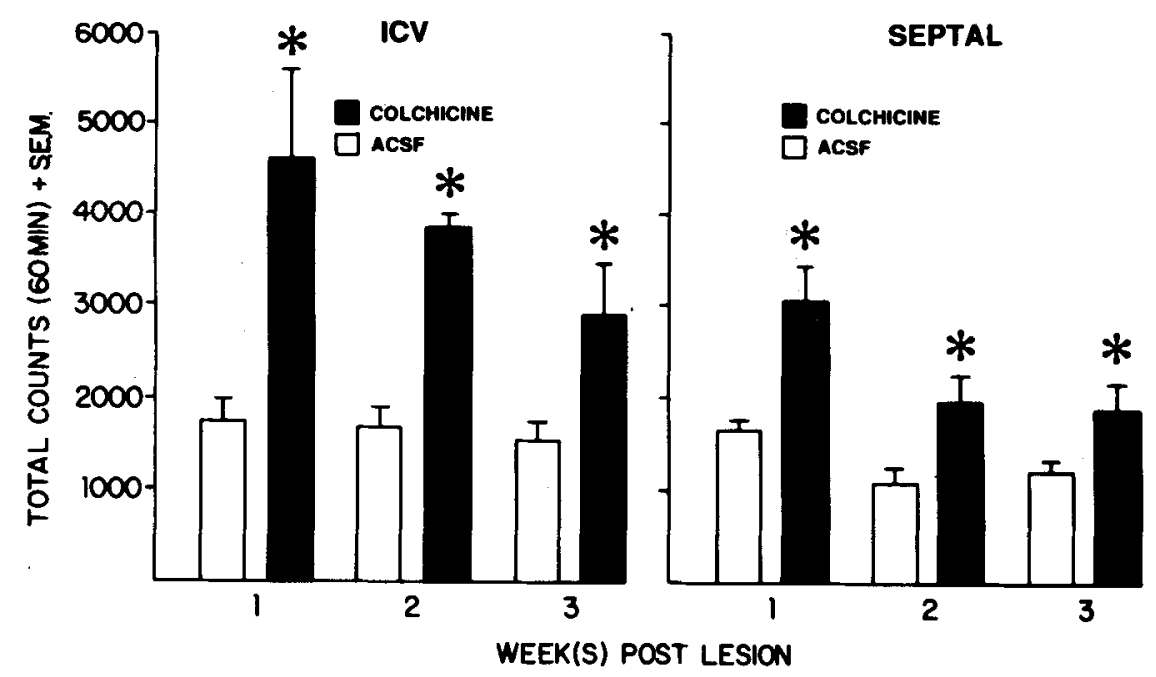

Figure 1. Effects of colchicine on locomotor activity 1,2 , and 3 weeks after intracerebroventricular (ICV) or direct injection into the medial septum. Data are average counts/60 min $\pm S E M$ $(n=12$ rats per treatment). $p<.001$ versus respective routes of ACSF administration. 
the rats receiving colchicine by the ICV route of administration indicated a significant treatment effect $[F(1,12)=$ $21.03, p<.0006]$, but the week effect $[F(2,24)=2.94$, $p>.0963]$ and the week $\times$ treatment interaction $[F(2,24)=1.70, p>.2146]$ were not significant. A repeated measures ANOVA of the data from the animals receiving direct administration of colchicine in the medial septum indicated a significant treatment effect $[F(1,14)=$ $16.88, p<.0011]$. The week effect was significant $[F(2,28)=6.76, p<.0104]$, but the week $\times$ treatment interaction was not $[F(2,28)=0.97, p>.3923]$. These data indicate that, by either route of administration of colchicine, motor activity was increased significantly for up to 3 weeks. To directly compare the hyperactivity resulting from ICV or direct septal administration of colchicine, data from the third week were transformed to percent of respective vehicle controls. Analysis of the transformed data indicated that the measure of hyperactivity at 3 weeks was not statistically different between the two lesion groups $(p<.05$, Student's $t$ test).

\section{Morris Water Maze}

Administration of colchicine ICV had a significant effect on acquisition in the water maze (Figure 2, top panel). A repeated measures ANOVA indicated a significant treatment effect $[F(1,12)=8.96, p<.01]$, day effect $[F(8,96)$ $=17.96, p<.0001]$, and treatment $\times$ day interaction $[F(8,96)=2.60, p<.05]$. Significant differences between the treated and the control rats were observed start-

ICV
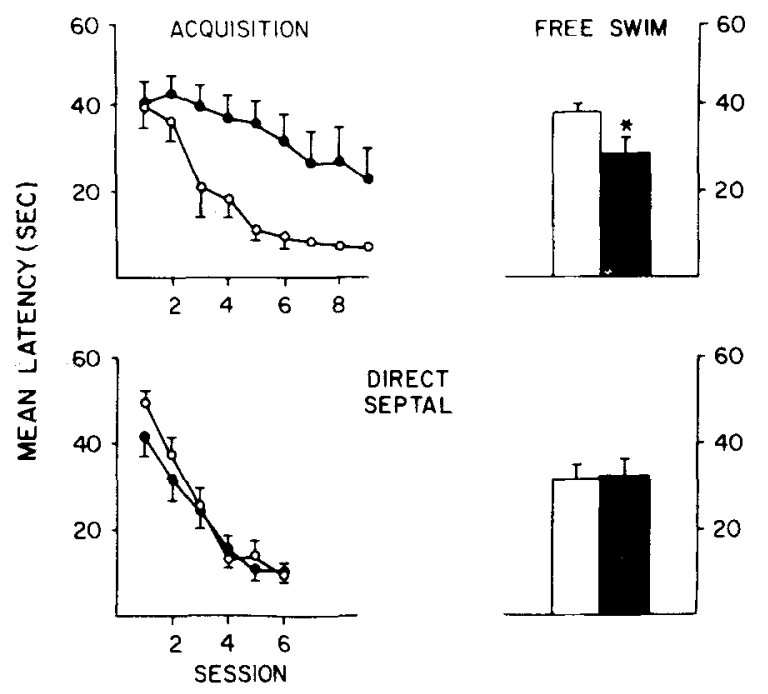

Figure 2. Effects of colchicine on acquisition in the water maze. Rats were given colchicine by intracerebroventricular infusion (ICV, top panel) or by direct injection into the medial septum (bottom panel) and tested beginning 4 weeks after injection. Data are average latencies ( $\sec \pm S E M$ ) to locate the platform ( $n=12$ rats per treatment). The black circles represent colchicine-treated rats; the open circles represent controls. To the right of latency graphs are the free-swim data. The black bars represent colchicine-treated rats and white bar controls. The ICV colchicine-treated rats spent significantly less time in the training quadrant $\left({ }^{*} p<.02\right)$; direct administration of colchicine had no effect on this measure of spatial learning $(p>.05)$.

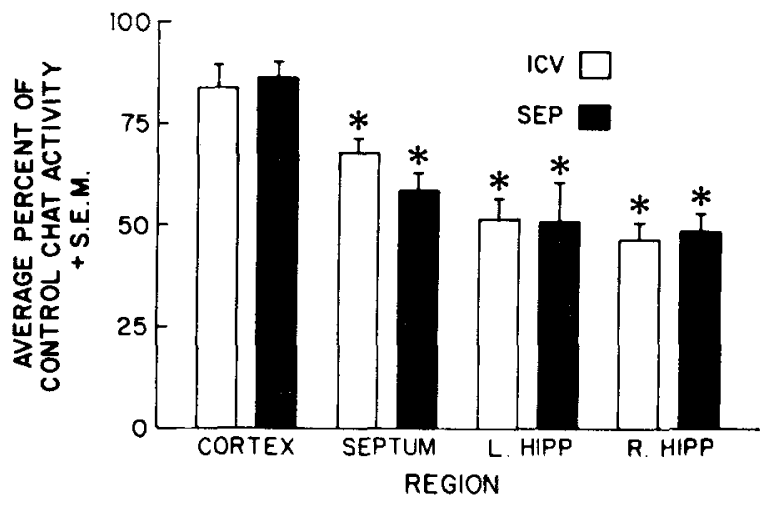

Figure 3. Effects of colchicine on ChAT levels in the septum, frontal cortex, and right or left hippocampus 5 weeks after injection. Data are expressed as average percent of control with 6 animals per treatment. Assays were performed at different times and are expressed as percent of control values. Control values for ICV infusions were ( $\mathrm{nmol} / \mathrm{h} / \mathrm{mg}$ protein $\pm S E M)$ : septum, $55.9 \pm 2.5$; frontal cortex, 58.1 \pm 9.1 ; left hippocampus, 73.0 44.6 ; right hippocampus, $52.8 \pm 2.6)\left({ }^{*} p<.05\right)$. Control values for the direct injection were (nmol/h/mg protein $\pm S E M$ ): septum, $48.0 \pm 1.0$; frontal cortex, $52.3 \pm 5.1$; left hippocampus, $41.4 \pm 3.6$; right hippocampus, $42.9 \pm 1.4$ $\left({ }^{*} p<.05\right)$.

ing on Day 3 of acquisition. In the posttraining freeswim test in which the platform was removed, the ICV colchicine-treated rats spent significantly less time $(27.6 \pm 4.0 \mathrm{sec})$ in the training quadrant than did the controls $(37.0 \pm 2.0 \mathrm{sec} ; t=2.3, p<.02)$. A repeated measures ANOVA of the data from the animals receiving colchicine directly into the medial septum indicated no significant treatment $(F=0.35)$ or day $\times$ treatment interaction ( $F=0.75$; see Figure 2 , bottom panel). There was a significant day effect $[F(5,70)=43.13, p<.0001]$. Direct colchicine treatment had no significant effect on the time $(32.8 \pm 4.9 \mathrm{sec})$ spent in the training quadrant, relative to controls $(32.4 \pm 3.9 \mathrm{sec})$, during the posttraining free-swim test $(p>.05)$.

\section{Neurochemistry}

The effects of ICV or direct administration of colchicine on ChAT activity are shown in Figure 3. Bilateral ICV administration significantly decreased ChAT activity in the septum by approximately $30 \%(p<.001)$, while direct administration significantly decreased ChAT activity in the septum by $40 \%(p<.001)$. The reduction of ChAT activity in the septum by the two routes of administration was not significantly different. There was a significant $50 \%$ decrease in ChAT activity in both right and left hippocampi, regardless of the route of administration $(p<.005)$. There was no significant effect of colchicine on ChAT activity in the frontal cortex (a decrease of $16 \%$ for ICV administration and $12 \%$ for direct injection), regardless of route of administration.

\section{Neuroanatomical Assessment}

Nissl-stained sections from the animals receiving colchicine by either route of administration indicated little 

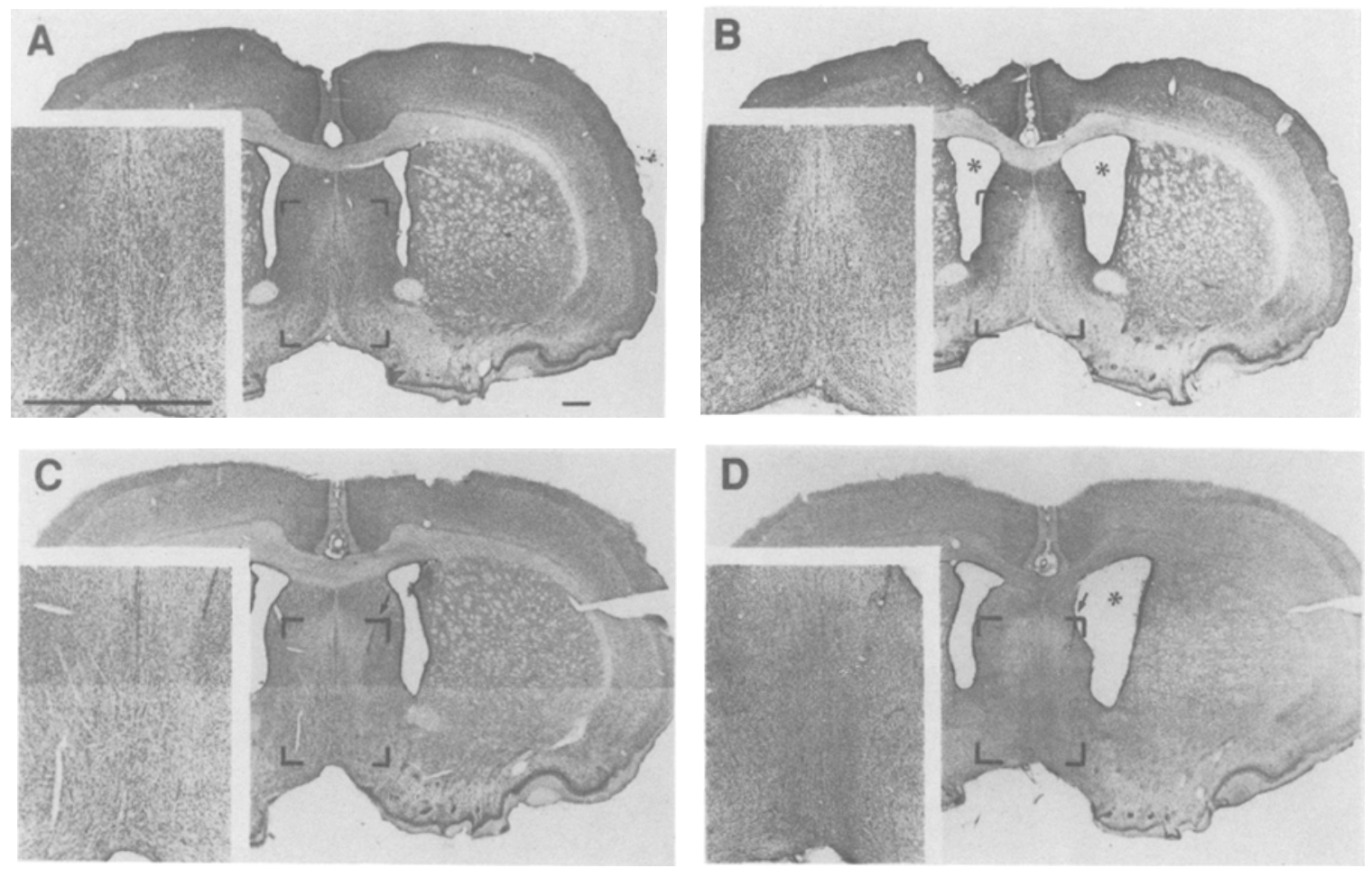

Figure 4. Histological evaluation of the effects of colchicine administration. Photomicrographs from 0.1\% thionin-stained coronal sections through the medial septum showing the general morphology of the area. (A) Control rat after ACSF ICV. (B) After bilateral injection of $3.75 \mu g$ colchicine ICV. Note bilateral shrinkage of septum and bilaterally enlarged ventricles (*). (C) Control rat after direct ACSF infusion. Arrow indicates needle path. (D) After 5 mg colchicine directly into the medial septum. Arrow indicates needle path. Note that shrinkage of lateral septum is unilateral and ventricle is enlarged ipsilateral to the injection (*). Bar $=500 \mu$.

detectable cell loss in the medial septum (Figure 4). Colchicine given either by ICV or directly into the medial septum caused shrinkage of the septal neuropil and a concomitant increase in the size of one or both of the lateral ventricles. In the animals receiving direct administration of colchicine into the medial septum, the ipsilateral ventricle was larger than the contralateral ventricle. In the animals receiving ICV colchicine, both lateral ventricles were enlarged, with a concomitant shrinkage of the lateral septum. Nissl-stained sections of the hippocampus showed no appreciable cell loss in the pyramidal cell layer or the dentate gyrus after either colchicine treatment (Figure 5).

The effect of colchicine on ChAT IR cell bodies in the medial septum is shown in Figure 6 and Table 1. A threeway ANOVA indicated that colchicine injections significantly reduced the number of ChAT IR cell bodies [effect of treatment, $F(1,44)=235.6, p<.0001$; treatment $\times$ method of injection interaction, $F(1,44)=7.6, p<$ $.01]$. Further analysis showed that both bilateral ICV administration (Figure 6B) and direct infusion into the medial septum (Figure 6C) resulted in a significant reduction of ChAT IR cells, relative to controls $(p<.0001)$. However, the pattern of cell loss was different for the two methods of injection [significant effect of side, $F(1,44)=$ $21.3, p<.0001$; side $\times$ method of injection interaction,
$F(1,44)=9.7, p<.01]$. Univariate analysis indicated that cell loss was significantly greater on the right side (ipsilateral to the injection) than on the left side for both control and colchicine-treated rats after direct administration into the medial septum $(p<.0001)$. Further analysis indicated that direct septal injections of the vehicle alone, relative to ICV vehicle injections, significantly reduced the number of ChAT IR cell bodies in both sides of the medial septum $(p<.0001)$. However, the loss after direct vehicle injections was significantly less than after direct colchicine injections $(p<.0001)$. Both routes of administration spared ChAT IR cells in the vertical limb of the diagonal band (data not shown).

Coronal sections of the hippocampal formation stained histochemically for $\mathrm{AChE}$ revealed a difference between ACSF infusion into the ventricles and ACSF infusion directly into the medial septum (Figure 7), with the direct infusion of vehicle resulting in a slight, but consistent, decrease in AChE staining in the outer two thirds of the molecular layer of the dentate gyrus. Both routes of colchicine administration, either bilateral colchicine ICV $(3.75 \mu \mathrm{g} /$ side; Figure $7 \mathrm{~B})$ or direct administration $(5.0 \mu \mathrm{g})$; (Figure 7C) into the medial septum resulted in a marked decrease in AChE staining in the dentate gyrus, especially in the outer two thirds of the molecular layer of the den- 

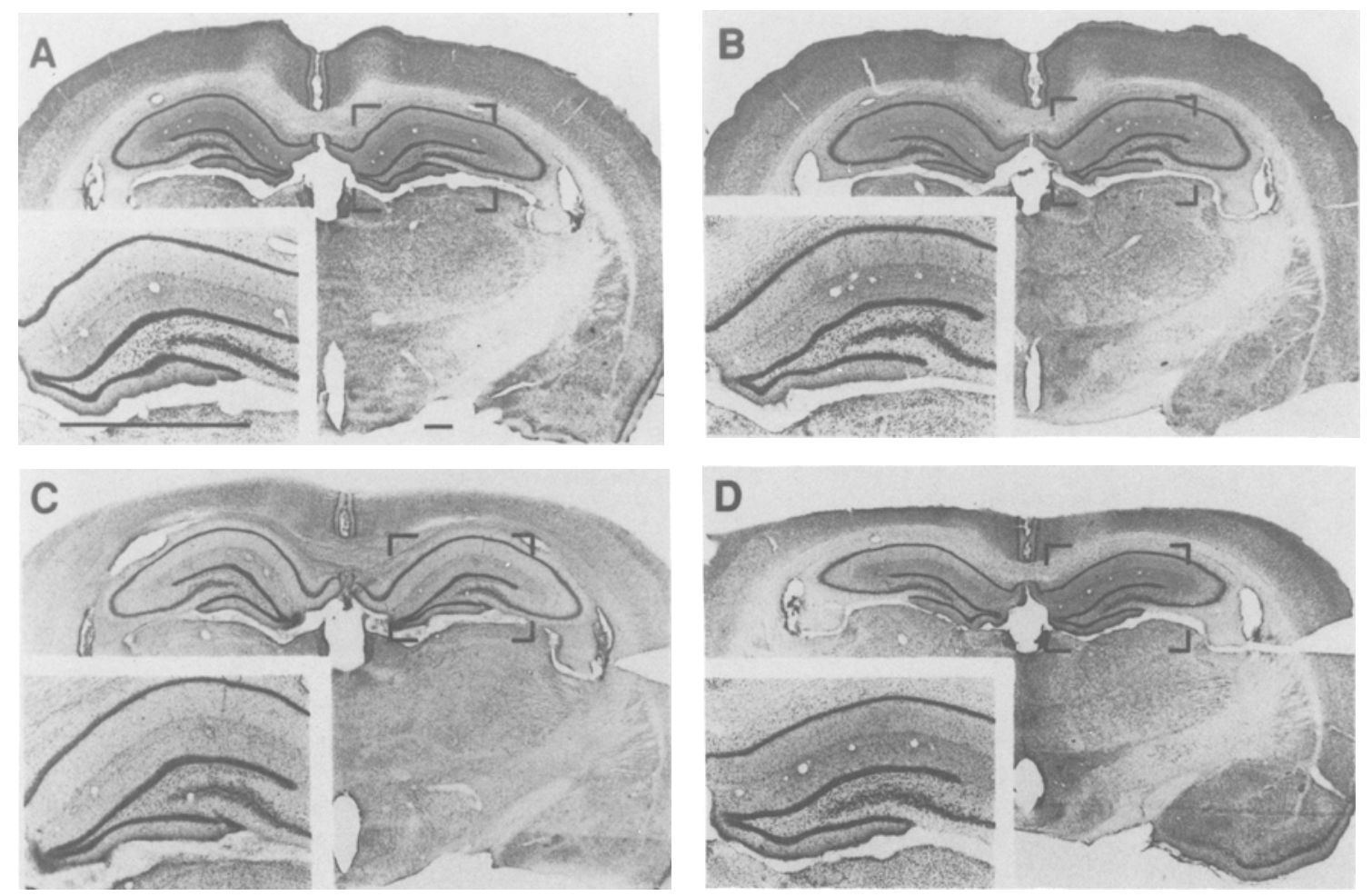

Figure 5. Histological evaluation of the effects of ICV or direct septal colchicine administration on $0.1 \%$ thionin-stained coronal sections of dorsal hippocampus (insets are higher magnification of right dentate gyrus). (A) Control rat after ACSF ICV. (B) After $3.75 \mathrm{mg}$ colchicine bilaterally ICV. No cell loss is discernible in Niss-stained sections. (C) After direct ACSF infusion into the medial septum. (D) After $5 \mu \mathrm{g}$ colchicine directly into the medial septum. No cell loss is discernible in Nissl-stained sections. Bar $=500 \mu$.

tate gyrus. However, this decrease in AChE staining was less uniform and generally less severe in the contralateral than in the ipsilateral dentate gyrus following direct administration of colchicine (Figure 7).

\section{DISCUSSION}

These tests demonstrated that ICV or direct infusion of colchicine into the medial septum produced increased locomotor activity, an effect reported by other laboratories in animals with lesions in the septum (Gray \& McNaughton, 1983). Colchicine given ICV also inter-

Table 1

Number of ChAT Immunoreactive Cell Bodies in the Medial Septum

\begin{tabular}{lccccccc} 
& \multicolumn{5}{c}{ Treatment } \\
\cline { 2 - 4 } \cline { 5 - 7 } Side & \multicolumn{3}{c}{ ICV } & \multicolumn{3}{c}{ MS-Direct } \\
\cline { 2 - 6 } \cline { 5 - 7 } Left & 107.0 & Colchicine & $\%$ & & Vehicle & Colchicine & $\%$ \\
& \pm 4.3 & $\pm 4.5^{*}$ & 45 & & $81.5 \dagger \ddagger$ & $45.8^{*} \dagger$ & 56 \\
Right & 103.5 & $44.8^{*}$ & 43 & & $68.3 \ddagger$ & $22.3^{*}$ & 33 \\
& \pm 3.7 & \pm 3.8 & & \pm 3.8 & \pm 3.8 & \\
\hline
\end{tabular}

Note-Percentages reflect ratio of ChAT IR cell bodies in colchicinetreated versus vehicle-injected controls for a given side. *Significantly less than respective vehicle injection $(p<.0001)$. †Significantly greater than right side of medial septal direct infusions $(p<.0001)$. $\ddagger$ Significantly less than ICV vehicle injections $(p<.001)$. fered with the acquisition of a trial-independent, reference memory task in the water maze, an effect reported by other laboratories using different lesion techniques (Hagan et al., 1988; Kelsey \& Landry, 1988; Miyamoto et al., 1987; Nilsson et al., 1988). However, direct administration of colchicine into the medial septum had no effect on the ability of the rats to acquire a spatial memory task in the water maze. This difference in water-maze escape latency between the two colchicine-treated groups was supported by the free-swim data, which provides an additional assessment of spatial learning that is not confounded by nonspecific effects on performance or locomotor activity.

It was also observed that both colchicine treatments decreased ChAT levels in both sides of the hippocampus with little effect on ChAT levels in the frontal cortex, effects also reported by other laboratories using different lesion procedures (Hagan et al., 1988; Miyamoto et al., 1987; Nilsson et al., 1988). In addition, both colchicine treatments decreased ChAT levels in the septum to the same extent.

These neurochemical changes in the septum and hippocampus were associated with an obvious loss of ChAT IR neurons in the septum in the absence of discernible cell loss in the medial septum by Nissl-staining, consistent with the report by Peterson and McGinty (1988) after ICV injection of colchicine. Thus, when given either 

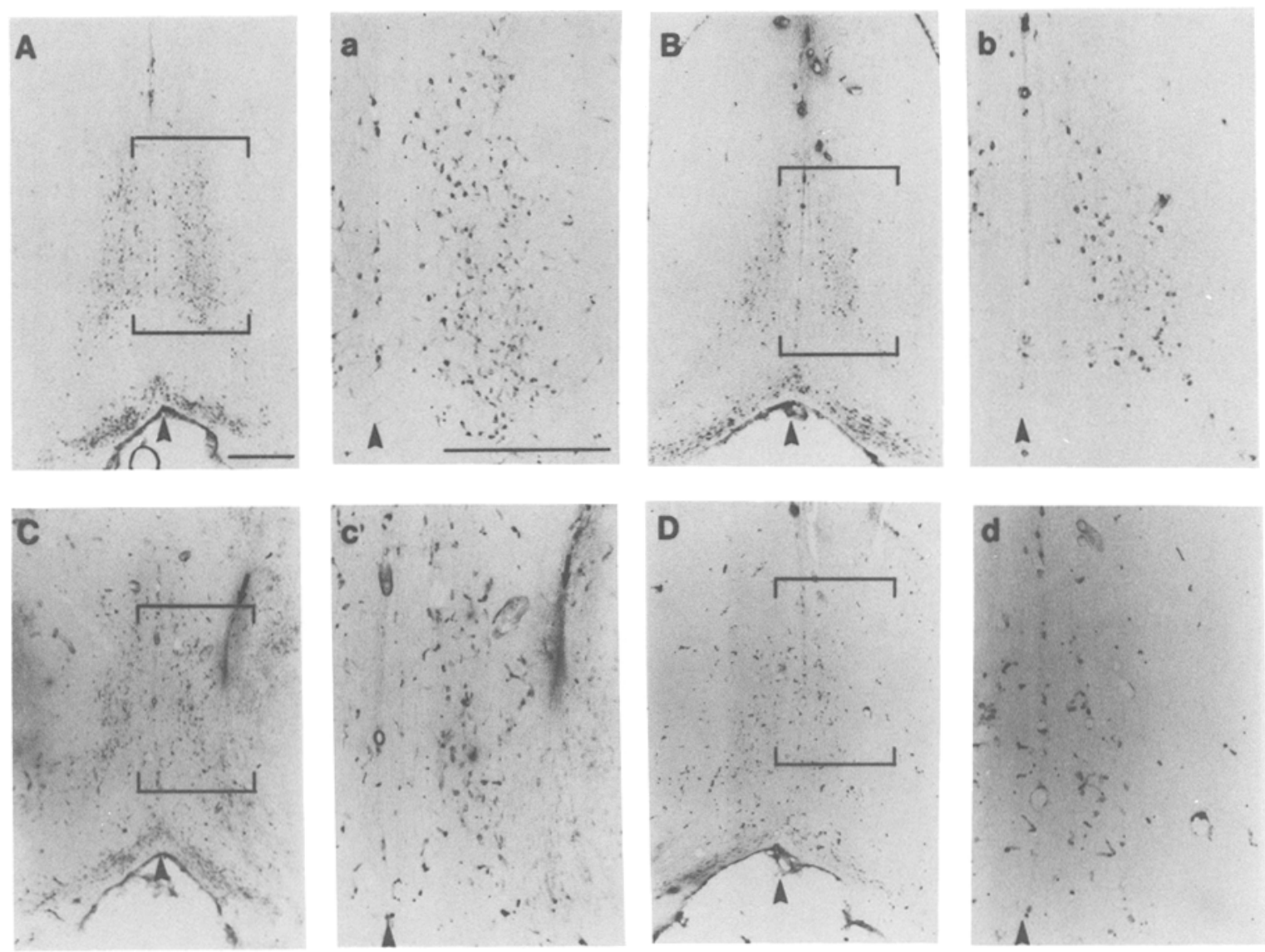

\begin{abstract}
Figure 6. Histological evaluation of the effects of colchicine administration. Photomicrographs from coronal sections stained immunocytochemically for ChAT showing presence of ChAT immunoreactive cell bodies in the medial septum. (A) Control rat after ACSF ICV. Area contained within box shown at higher magnification in a. (B) After bilateral injection of 3.75 colchicine ICV. Area contained within box shown at higher magnification in b. (C) After direct ACSF infusion. Area contained within box shown at higher magnification in c. (D) After $5 \mu$ colchicine directly into the medial septum. Area contained within box shown at higher magnification in d. Note decrease in number of ChAT immunoreactive cell bodies after both routes of colchicine administration. Arrows indicate direct injection site. Arrow heads denote midline in all pictures. Bar $=500 \mu$.
\end{abstract}

ICV or directly, colchicine produces neurochemical and neuroanatomical effects similar to those reported following lesions of the septum using electrical current (Kelsey \& Landry, 1988; Miyamoto et al., 1987), ibotenic acid (Hagan et al., 1988), and radio frequency (Nilsson et al., 1988), but without the massive tissue destruction characteristic of these other lesions. However, the effects of the two routes of colchicine administration employed in this study were not identical. The direct administration of colchicine resulted in a greater decrease in ChAT immunoreactivity on the right side than on the left side of the medial septum. This differed from ICV administration of colchicine in which there was no side effect on the number of ChAT IR cells. In addition, the vehicle injection directly into the medial septum resulted in a significant decrease in the number of ChAT IR cells, relative to the ICV vehicle injections.

The finding that direct administration of colchicine did not result in a water-maze deficit was surprising since neurochemical measures of ChAT activity in the right and left hippocampus in both lesion paradigms of this study were quantitatively similar. These decrements parallel previously reported decreases in hippocampal ChAT activity ranging from $55 \%$ to $85 \%$ of controls after medial septal lesions that were associated with deficits in reference memory tasks (Hagan et al., 1988; Miyamoto et al., 1987; Nilsson et al., 1988). One interpretation of previous work indicating that septal lesions interfere with acquisition in the water maze is that the lesion procedure may have affected the fimbria-fornix or damaged neurochemical systems passing through or adjacent to the medial septum and therefore may not represent a selective disruption of the septohippocampal cholinergic pathway. The two studies using electrolytic lesions of the medial septum (Kelsey \& Landry, 1988; Miyamoto et al., 1987) both showed a spread of the lesion to include the fimbriafornix. In the study by Nilsson et al. (1988), radio frequency was used to lesion the medial septum; no histology was reported, but it was indicated that medial septal lesions caused a $50 \%-75 \%$ decrease in serotonin levels 

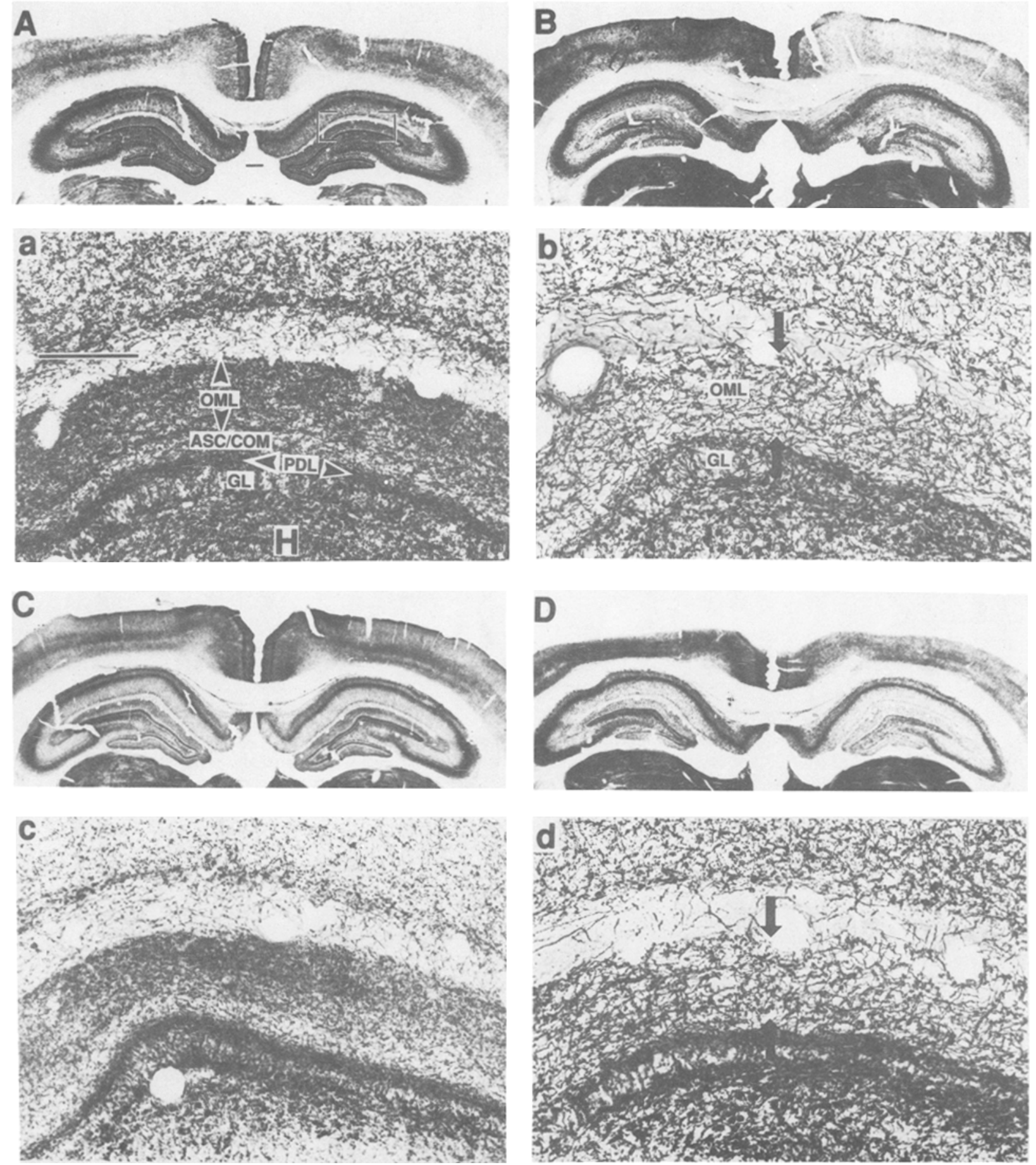

Figure 7. Histological evaluation of the effects of colchicine administration. Photomicrographs from hippocampal coronal sections stained histochemically for acetylcholine esterase (AChE). (A) Control rat after infusion of ACSF ICV. (a) Ipsilateral dentate gyrus of control rat after infusion ACSF ICV (H = hilus; GL = granule cell layer; $P D L=$ proximal dendritic layer; $A S C / C O M=$ asscociational commisural layer; $\mathrm{OML}=$ outer molecular layer). (B) After bilateral infusion of $3.75 \mu \mathrm{g}$ colchicine ICV. (b) Ipsilateral dentate gyrus after bilateral infusion of 3.75 $\mu \mathrm{g}$ colchicine ICV vehicle. (C) After direct ACSF infusion. (c) Ipsilateral dentate gyrus after direct ACSF infusion of vehicle. (D) After direct infusion of $5 \mu \mathrm{g}$ colchicine into the medial septum. (d) Ipsilateral dentate gyrus after direct infusion $5 \mu \mathrm{g}$ colchicine into the medial septum. Note dramatic decrease in staining in the outer two thirds of the molecular layer (OML) of the dentate gyrus following both routes of colchicine administration (b and d; between arrows). Bar $=200 \mu$. 
in the hippocampus, a distinct projection system. Hagan et al. (1988) injected ibotenic acid into the medial septum, reporting destruction of cell bodies in the medial septum detectable by Nissl staining. Although ibotenic acid is generally believed to destroy cell bodies preferentially, leaving fibers of passage intact, there is a risk that the resultant neurotoxicity could have produced a hole in the tissue proximal to the injection site or spread to other areas and cell types.

The results of the present experiments support the hypothesis that spatial learning deficits observed after lesions of the medial septum are not solely due to disruption of the septohippocampal cholinergic pathway. The neuroanatomical and neurochemical effects of colchicine administered by the two routes were similar, yet directly administered colchicine did not interfere with learning, whereas ICV colchicine effectively disrupted acquisition. One interpretation of this finding is that directly administered colchicine resulted in a more focused lesion in the area of the medial septum affecting the ipsilateral side more than the contralateral side. That the lesion was focal in nature is supported by the AChE staining of the hippocampal formation in which only fibers projecting to the dentate gyrus appeared to be significantly affected by the lesion at the time examined. Furthermore, the colchicine-induced effect on AChE staining on the ipsilateral side was more pronounced with direct than with ICV administration, suggesting that (1) the behavioral outcome of the two lesion paradigms examined herein may be the result of the anterior to posterior heterogeneity of the cholinergic populations affected by the two procedures and (2) ICV injections resulted in more effective bilateral lesions of the septum. However, another explanation is that ICV colchicine administration may have spread to areas other than the medial septum and affected noncholinergic neurotransmitter systems by diffusing from the ventricles through the lateral septum to affect the medial septum, thus leading to the learning deficit seen in the water maze. This is consistent with observations of Nissl-stained material, which indicated that the lateral septum was affected after both routes of administration; however, this effect was only bilateral after ICV infusion of colchicine.

Finally, the learning deficit seen after ICV and not direct administration of colchicine may be a consequence of longterm disruption of axoplasmic transport in projections of the fornix containing multiple transmitter systems. Peterson and McGinty (1988) reported that unilateral ICV colchicine appeared to have specific neurotoxic effects on cholinergic neurons, sparing GABAergic neurons in the medial septum. However, there was evidence for the spread of colchicine into the medial caudate surrounding the lateral ventricle in their study. Lateral to the injection site, Peterson and McGinty observed a medial to lateral gradient of cholinergic cell loss in the striatum; GABAergic neurons in the striatum appeared normal. It was not possible to determine the extent to which colchicine may have spread to other structures or affected noncholinergic neurotrans- mitter systems in the present study. However, investigation of colchicine's effects on other neurotransmitter systems is planned in conjunction with morphometric analysis of ChAT immunoreactivity in the medial septum after direct and ICV administration of colchicine.

\section{REFERENCES}

Bartus, R. T., Flicker, C., Dean, R. L., Pontecorvo, M., Figueriredo, J. C., \& Fisher, S. K. (1985). Selective memory loss following nucleus basalis lesions: Long-term behavioral recovery despite persistent cholinergic deficiencies. Pharmacology, Biochemistry \& Behavior, 23, 125-135.

Becker, J. T., WAlker, J. A., \& Olton, D. S. (1980). Neuroanatomical bases of spatial memory. Brain Research, 200, 307-320,

Bigl, V., Woolf, N. J., \& BuTcher, L. L. (1982). Cholinergic projections from the basal forebrain to frontal, parietal, temporal, occipital, and cingulate cortices combined fluorescent traces and acetylchonesterase analysis. Brain Research Bulletin, 8, 727-749.

BRADFord, M. (1976). A rapid and sensitive method for the quantitation of microgram quantities of protein utilizing the principle of proteindye binding. Analytical Biochemistry, 72, 248-254.

Crutcher, K. A., Kesner, R. P., Novak, J. M. (1983). Medial septal lesions, radial arm maze performance, and sympathetic sprouting: A study of recovery of function. Brain Research, 262, 91-98.

Eckenstein, F., \& Thoenen, H. (1982). Production of specific antisera and monoclonal antibodies to choline acetyltransferase: Characterization and use for identification of cholinergic neurons. $E M B O$ Joumal, 1, 363-368.

Fibiger, H. C. (1982). The organization and some projections of cholinergic neurons of the mammalian forebrain. Brain Research Review, 4, 327-388.

Fonnum, F. (1975). A rapid radiochemical method for the determination of acetyltransferase. Joumal of Neurochemistry, 24, 407-409

FreUND, T. F., a ANTAL, M. (1988). GABA-containing neurons in the septum control inhibitory interneurons in the hippocampus. $\mathrm{Na}$ ture, 336, 170-173.

Geisser, S., Greenhouse, S. W. (1958). An extension of Box's results on the use of the $\mathrm{F}$ distribution in multivariate analysis. Annals of Mathemathetical Statistics, 29, 885-891.

GlowinskI, J., \& IVERSEN, L. L. (1966). Regional studies of catecholamines in the rat brain: 1 . The disposition of $3 \mathrm{H}$-norepinephrine and $3 \mathrm{H}-\mathrm{DOPA}$ in various regions of the brain. Joumal of Neurochemistry, 13, 655-669.

Gray, J. A., \& McNaughton, N. (1983). Comparison between the behavioral effects of septal and hippocampal lesions: A review. Neuroscience \& Biobehavioral Reviews, 7, 119-188.

Greenhouse, S. W., Geisser, S. (1959). On methods in the anal ysis of profile data. Psychometrika, 24, 95-112.

Hagan, J. J., Salamone, J. D., Simpson, J., Iversen, S. D., \& Morris, R. G. M. (1988). Place navigation in rats is impaired by lesions of medial septum and diagonal band but not nucleus basalis magnocellularis. Behavioral Brain Research, 27, 9-20.

Hedreen, J. C., Bacon, S. J., \& Price, D. L. (1985). A modified histochemical technique to visualize acetylcholinesterase-containing axons. Journal of Histochemistry \& Cytochemistry, 33, 134-140.

Hsu, S.-M, RAINE, L., \& FANGER, H. (1981). Use of avidin-biotin peroxidase complex $(A B C)$ in immunoperoxidase techniques: $A$ comparison between $A B C$ and unlabeled antibody (PAP) procedures. Journal of Histochemistry \& Cytochemistry, 29, 577-580.

Johnston, M., McKinney, M., \& Coyle, J. (1979). Evidence for a cholinergic projection to neocortex from neurons in basal forebrain. Proceedings of the National Academy of Science, 76, 5392-5396.

Kelsey, J. E., LandRY, B. A. (1988). Medial septal lesions disrupt mapping ability in rats. Behavioral Neuroscience, 102, 289-293.

Knowlton, B. J., Wenk, G. L., Olton, D. S., \& Coyle, J. T. (1985). Basal forebrain lesions produce a dissociation of trial-dependent and trial-independent memory performance. Brain Research, 345, 315-321. Kohler, C., Chan-Palay, V., Jang-Yen, W. U. (1984). Septal 
neurons containing glutamic acid decarboxylase immunoreactivity project to the hippocampal region in the rat brain. Anatomy \& Embryology, 169, 41-44.

Lehman, J., Nagy, J. I., Almadja, S., \& Fibiger, H. C. (1980). The nucleus basalis magnocellularis: The origin of a cholinergic projection to the neocortex of the rat. Neurosciences, 5, 1161-1174.

Miyamoto, M., Kato, J., Narumi, S., \& Nagaoka, A. (1987). Characteristics of memory impairment following lesioning of the basal forebrain and medial septal nucleus in rats. Brain Research, 419, 19-31.

MundY, W. R., \& TiLson, H. A. (1988). Behavioral impairment in the rat after colchicine lesions of the hippocampus and nucleus basalis. Neurotoxicology, 9, 511-520.

Nilsson, O. G., Shaptro, M. L., Olton, D. S., Gage, F. H., \& BuorkLUND, A. (1987). Spatial learning and memory following fimbriafornix transection and grafting of fetal septal neurons to the hippocampus. Experimental Brain Research, 67, 195-215.

Nilsson, O. G., Strecker, R. E., Daszuta, A., \& Bjorklund, A. (1988). Combined cholinergic and serotonergic denervation of the forebrain produces severe deficits in a spatial leaming task in the rat. Brain Research, 453, 235-246.

Olton, D. S., Walker, J. A., \&age, F. H. (1978). Hippocampal connection and spatial discrimination. Brain Research, 139, 295-308.

Peterson, G. M. (1989). A quantitative analysis of the crossed septohippocampal projection in the rat. Anatomy \& Embryology, 180, $421-425$.

Peterson, G. M., \& McGinty, J. F. (1988). Direct neurotoxic effects of colchicine on cholinergic neurons in medial septum and striatum. Neuroscience Letters, 94, 46-51.

Sutherland, R. J., \& RodrigueZ, A. J. (1989). The role of the fornix/fimbria and some related subcortical structures in place learning and memory. Behavioral Brain Research, 32, 265-277.

Swanson, L. W., \& Cowan, W. M. (1979). The connections of the septal region in the rat. Journal of Comparative Neurology, 186, 621-656.

Tilson, H. A., Mclamb, R. C., Shaw, S., Rogers, B. C., PediADITAKIs, P., CoOK, L. (1988). Radial-arm maze deficits produced by colchicine administered into the area of the nucleus basalis are ameliorated by cholinergic agents. Brain Research, 54, 45-52.

Wainer, B. H., Levey, A. I., Rye, D. B., Mesulam, M., \& Mufson, E. J. (1985). Cholinergic and noncholinergic septohippocampal pathways. Neuroscience Letters, 54, 45-52.

Whitehouse, P. J., Price, D. L., Strubie, R. G., Clerk, A. W., Coyle, J. T., Delong, M. R. (1982). Alzheimer's disease and senile dementia: Loss of neurons in the basal forebrain. Science, $\mathbf{2 1 5}$, 1237-1239.

Woolf, N. J., Eckenstein, F., \& Butcher, L. L. (1984). Cholinergic systems in the rat brain: I. Projections to the Limbic Telencephalon. Brain Research Bulletin, 13, 751-784.

(Manuscript received September 8, 1989; revision accepted for publication October 16, 1990.) 